\title{
False positivity of Rose Bengal test in patients with COVID-19: case series, uncontrolled longitudinal study
}

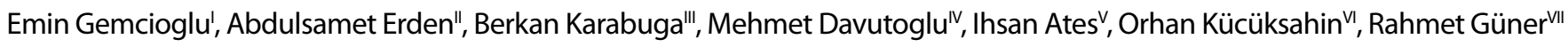

Ankara City Hospital, Ankara, Turkey

'MD. Physician, Department of Internal Medicine, Ankara City Hospital, Ankara, Turkey.

(D) orcid.org/0000-0001-9751-8452

"MD. Physician, Division of Rheumatology, Department of Internal Medicine, Ankara City Hospital, Ankara, Turkey.

(D) orcid.org/0000-0002-8084-2018

'"MD. Resident, Department of Internal Medicine, Ankara City Hospital, Ankara, Turkey.

(D) orcid.org/0000-0001-5357-7610

"MD. Resident, Department of Internal Medicine, Ankara City Hospital, Ankara, Turkey.

(D) orcid.org/0000-0001-9618-8885

'MD. Associate Professor, Department of Internal Medicine, Ankara City Hospital, Ankara, Turkey.

(D) orcid.org/0000-0003-2858-6229

"MD. Associate Professor, Division of Rheumatology, Department of Internal Medicine, Ankara City Hospital, Ankara, Turkey.

(D) orcid.org/0000-0003-4530-2304

"IIMD. Professor, Department of Infectious Diseases and Clinical Microbiology, Ankara City Hospital, Ankara, Turkey.

(D) orcid.org/0000-0002-1029-1185
Dear Editor

At the end of 2019, a novel coronavirus was identified as the cause of a cluster of pneumonia cases in Wuhan, China, and it spread quickly to other countries. This has led to a pandemic that has spread throughout most countries of the world in 2020. A wide variety of symptoms and signs can be seen in coronavirus disease 2019 (COVID-19) infection, such as fever, coughing, shortness of breath, arthralgia, muscle pain and diarrhea.

COVID-19 infection may show clinical or laboratory features that are similar to those of a variety of diseases. For example, it is difficult to distinguish dengue and COVID-19 because they have shared clinical and laboratory features. ${ }^{1,2}$ In a case report from Singapore, two patients with false-positive results from rapid serological testing for dengue, who were later confirmed to have severe acute respiratory syndrome COVID-19 infection, were reported. ${ }^{3}$

Brucellosis is the most common zoonosis worldwide and is a significant public health problem in many developing countries such as our country, Turkey. ${ }^{4}$ Brucellosis typically presents with fever, malaise and arthralgia. ${ }^{5}$ Common symptoms of COVID-19, such as fever, myalgia or arthralgia, can also be seen in brucellosis. The laboratory findings of these infections may be similar. Thrombocytopenia and leukopenia are common in COVID-19 and can also be seen in brucellosis. When there is a suspicion of brucellosis, the Rose Bengal test is recommended as the first test. This is a plaque agglutination test with high sensitivity that is easy to apply, has low cost and provides qualitative results. Therefore, it is frequently used as a screening test in human brucellosis cases. For this reason, we performed the Rose Bengal test on patients with COVID-19 presenting fever and arthralgia, because our country is within the endemic region for brucellosis.

In our tertiary-level medical facility, the patients received their diagnoses either through a positive polymerase chain reaction (PCR) for SARS-CoV-2 or through fulfilling three clinical criteria, including having fever and/or respiratory symptoms, compatible chest imaging findings and decreased lymphocyte count. ${ }^{6}$ We questioned the patients in our case series to ascertain any history of brucellosis, but found that none of them had any history relating to brucellosis or other zoonotic diseases. In eight of these patients, Rose Bengal tests were positive. These results are provided in Table $\mathbf{1}$. The median age of these eight patients was 58.5 years, and five of them were female. Arthralgia and fatigue were present in all eight patients; fever and cough were present in seven (87.5\%). No patient had anosmia, ageusia, abdominal pain or diarrhea. Fourteen days after treatment for COVID-19, we performed the Rose Bengal test on all patients again, and its positivity became negative.

The Rose Bengal test may sometimes give a false-positive result. Various antigens obtained from Brucella melitensis and Brucella abortus are generally used in serological tests. Among these, the most widely used antigen is smooth lipopolysaccharide (S-LPS). The Rose Bengal test detects S-LPS-specific immunoglobulin M (IgM), immunoglobulin G (IgG) and immunoglobulin A ( $\operatorname{IgA})$ antibodies. However, this test lacks specificity to discriminate the false-positive serological reactions caused by bacteria (especially Gram-negative bacteria) sharing S-LPS epitopes with Brucella. ${ }^{7,8}$

It has been shown that the Rose Bengal test might have cross-reactivity with certain bacteria, including are Francisella tularensis, Afipia, Escherichia hermannii, Stenotrophomonas maltophilia, Yersinia enterocolitica, Escherichia coli, Salmonella urbana, Vibrio cholerae and others. ${ }^{9-11}$ Other 
Table 1. Demographic and clinical characteristics and laboratory findings among eight patients with COVID-19

\begin{tabular}{|c|c|}
\hline Characteristic or finding & Value in patients $(n=8)$ \\
\hline Age - median (IQR) [years] & $58.5(47)$ \\
\hline Female sex - n (\%) & $5(62.5)$ \\
\hline COVID-19-n (\%) & $8(100)$ \\
\hline Duration of hospitalization - median (IQR) [days] & $8.5(5.25)$ \\
\hline Fatigue - n (\%) & $8(100)$ \\
\hline Arthralgia - $\mathrm{n}(\%)$ & $8(100)$ \\
\hline Fever - n (\%) & $7(87.5)$ \\
\hline Cough - n (\%) & $7(87.5)$ \\
\hline Myalgia - n (\%) & $4(50)$ \\
\hline Dyspnea - n (\%) & $3(37.5)$ \\
\hline Headache - n (\%) & $2(25)$ \\
\hline Back pain - n (\%) & $1(12.5)$ \\
\hline Total lymphocytes - median (IQR) [per $\mathrm{mm}^{3}$ ] & $1195(677.5)$ \\
\hline Lactate dehydrogenase - median (IQR) [U/liter] & $219.5(90.25)$ \\
\hline C-reactive protein - median (IQR) [mg/liter] & $24.5(89.5)$ \\
\hline Serum ferritin - median (IQR) [ $\mu \mathrm{g} /$ liter] & $80(155.75)$ \\
\hline Fibrinogen - median (IQR) [g/liter] & $3.49(2)$ \\
\hline D-dimer - median (IQR) [mg/liter] & $0.4(1)$ \\
\hline Positive Rose Bengal test - $\mathrm{n}(\%)$ & $8(100)$ \\
\hline
\end{tabular}

$\mathrm{IQR}=$ interquartile range.

false-positive reactions in the Rose Bengal test may be attributable to residual antibody activity from vaccinations, or to laboratory error. ${ }^{12}$

Our study is noteworthy in that it was the first case series to show cross-reactivity between the Rose Bengal test and the COVID19 PCR test. Further studies are needed to explain the relationship between COVID-19 and the Rose Bengal test.

Ethics approval for this study was obtained from the Ethics Committee of a local hospital (number: E1-20-895; date: June 10, 2020).

\section{REFERENCES}

1. Chen $N$, Zhou M, Dong $X$, et al. Epidemiological and clinical characteristics of 99 cases of 2019 novel coronavirus pneumonia in Wuhan, China: a descriptive study. Lancet. 2020;395(10223):507-13. PMID: 32007143; doi: 10.1016/S0140-6736(20)30211-7.

2. Yan G, Pang L, Cook AR, et al. Distinguishing Zika and dengue viruses through simple clinical assessment, Singapore. Emerg Infect Dis. 2018;24(8):1565-8. PMID: 30016242; doi: 10.3201/eid2408.171883.

3. Yan G, Lee CK, Lam LTM, et al. COVID-19 and false-positive dengue serology in Singapore. Lancet Infect Dis. 2020;20(5):536. PMID: 32145189; doi: 10.1016/S1473-3099(20)30158-4.

4. Pappas G, Akritidis N, Bosilkovski M, Tsianos E. Brucellosis. N Engl J Med. 2005;352(22):2325-36. PMID: 15930423; doi: 10.1056/NEJMra050570.

5. Bosilkovski M, Krteva L, Dimzova M, et al. Human brucellosis in Macedonia - 10 years of clinical experience in endemic region. Croat Med J. 2010;51 (4):327-36. PMID: 20718086; doi: 10.3325/cmj.2010.51.327.

6. Shen $\mathrm{KL}$, Yang $\mathrm{YH}$, Jiang RM, et al. Updated diagnosis, treatment and prevention of COVID-19 in children: experts' consensus statement (condensed version of the second edition). World J Pediatr. 2020;16(3):232-9. PMID: 32333248; doi: 10.1007/s12519-020-00362-4.

7. Muñoz PM, Blasco JM, Engel B, et al. Assessment of performance of selected serological tests for diagnosing brucellosis in pigs. Vet Immunol Immunopathol. 2012;146(2):150-8. PMID: 22445082; doi: 10.1016/j. vetimm.2012.02.012.

8. Corbel MJ. Brucellosis in humans and animals. Geneva: World Health Organization; 2006. Available from: https://www.who.int/csr/resources/ publications/Brucellosis.pdf?ua=1. Accessed in 2020 (Aug 25).

9. Young EJ. Human brucellosis. Rev Infect Dis. 1983;5(5):821-42. PMID: 6356268; doi: 10.1093/clinids/5.5.821.

10. DíazR, Casanova A, Ariza J,Moriyón I.The Rose BengalTest in Human Brucellosis: A Neglected Test for the Diagnosis of a Neglected Disease. PLoS Negl Trop Dis. 2011;5(4):e950. PMID: 21526218; doi: 10.1371/journal.pntd.0000950.

11. Mert A, Ozaras R, Tabak F, et al. The sensitivity and specificity of Brucella agglutination tests. Diagn Microbiol Infect Dis. 2003;46(4):241-3. PMID: 12944013; doi: 10.1016/s0732-8893(03)00081-6.

12. Chothe SK, Saxena HM. Innovative modifications to Rose Bengal plate test enhance its specificity, sensitivity and predictive value in the diagnosis of brucellosis. J Microbiol Methods. 2014;97:25-8. PMID: 24345764; doi: 10.1016/j.mimet.2013.12.005.

Authors' contributions: Gemcioglu E: investigation (equal), project administration (equal), writing-review and editing (equal) and analysis (equal); Erden A: data curation (equal), software (equal) and analysis (equal); Karabuga B: data curation (equal), visualization (equal) and writing-review and editing (equal); Davutoglu M: data curation (equal), visualization (equal) and writing-review and editing (equal); Ates I: writing-review and editing, validation (equal) and project administration (equal); Kücüksahin O: analysis (equal), validation (equal) and software (equal); and Güner R: supervision, writing-review and editing (equal) and investigation (equal). All authors approved the final version of the manuscript to be released

Sources of funding: The authors declare that this study did not receive any financial support

Conflicts of interest: The authors have no conflict of interest to declare

Date of first submission: August 28, 2020

Last received: August 28, 2020

Accepted: September 3, 2020

\section{Address for correspondence:}

Emin Gemcioglu

Department of Internal Medicine, Ankara City Hospital

Universiteler Mahallesi, 1604. Sokak, Number: 9, Cankaya - Ankara, Turkey

Tel. (+90) 5059369141

E-mail:egemcioglu@gmail.com 